\title{
Omega-3-Fettsäuren wirkungslos
}

Fragestellung: Kann mit der Gabe von Omega-3-Fettsäuren die Erkrankungsaktivität bei Patienten mit Multiple Sklerose (MS) reduziert werden?

Hintergrund: Die vermutliche Beeinflussbarkeit von Erkrankungen, insbesondere Autoimmunerkrankungen durch Nahrungsmittel bleibt ein für viele Patienten wichtiges Thema. In populär wissenschaftlichen Ratgebern wird immer wieder auf Omega-3-Fettsäure-haltige Nahrung verwiesen, da in einzelnen Fallberichten eine „immunmodulierende“ Beeinflussung verschiedener Erkrankungen, insbesondere autoimmunologischer Erkrankungen und der MS postuliert wurde.

Patienten und Methodik: Eingeschlossen wurden Patienten mit einer aktiven schubförmigen MS im Alter zwischen 18 und 55 Jahren mit einem EDSS-Score von 0-5,0. Insgesamt wurden 90 Patienten randomisiert und entweder mit Omega-3-Fettsäuren oder Placebo behandelt. Nach sechs Monaten wurden alle Patienten zusätzlich mit subkutanem Interferon-beta la (Rebif 44) dreimal pro Woche für weitere 18 Monate behandelt. Primäre Outcome-Kriterien waren die kumulative Zahl der neuen T1gewichteten gadoliniumanreichernden $\left(\mathrm{Gd}^{+}\right)$Herde innerhalb der ersten sechs Monate und die Zahl der neuen T1-gewichteten hypointensen Herde nach 24 Monaten. Während der Studie wurde jedoch entschieden, den zweiten Teil des primären Outcome-Kriteriums zu einem sekundären Outcome-Kriterium zu wandeln. Weitere sekundäre Outcome-Kriterien waren die kombinierte Gesamtaktivität (definiert als jede T1-gewichtete anreichernde Läsion und jede neue oder vergrößernde T2-Läsion in den ersten sechs Monaten und während der gesamten Studienzeit sowie die Zahl der Schübe während der ersten sechs Monate sowie der gesamten Untersuchungszeit).

Ergebnisse: Die demografischen Charakteristika waren in beiden Gruppen vergleichbar. In den ersten sechs Behandlungsmonaten zeigten sich keine signifikanten Unterschiede zwischen den beiden Gruppen ( Abb. 1). Auch nach 24 Monaten und der additiven Interferongabe ergaben sich für kein Outcome-Kriterium Abweichungen zwischen den beiden Gruppen. Im Hinblick auf die klinischen Parameter gab es ebenfalls keine Differenzen hinsichtlich der Zahl der erlittenen Schübe oder der Änderungen im EDSS-Score über den Zeitraum von sechs oder vierundzwanzig Monaten. Ebenso blieb die Zahl der berichteten Nebenwirkungen in beiden Gruppen identisch.

Schlussfolgerungen: Die Behandlung von Patienten mit schubförmiger MS mit Omega-3-Fettsäuren zeigt keinen Einfluss auf

Torkildsen O, Wergeland S, Bakke $S$ et al. Omega-3-fatty acid treatment in multiple sclerosis (OFAMS Study). Arch Neurol 2012; 69: 1044-51 kernspintomografische Parameter im Zeitraum von sechs Monaten. Bei MS-Patienten, die mit Interferon behandelt werden, haben Omega-3-Fettsäuren keinen additiven Effekt.

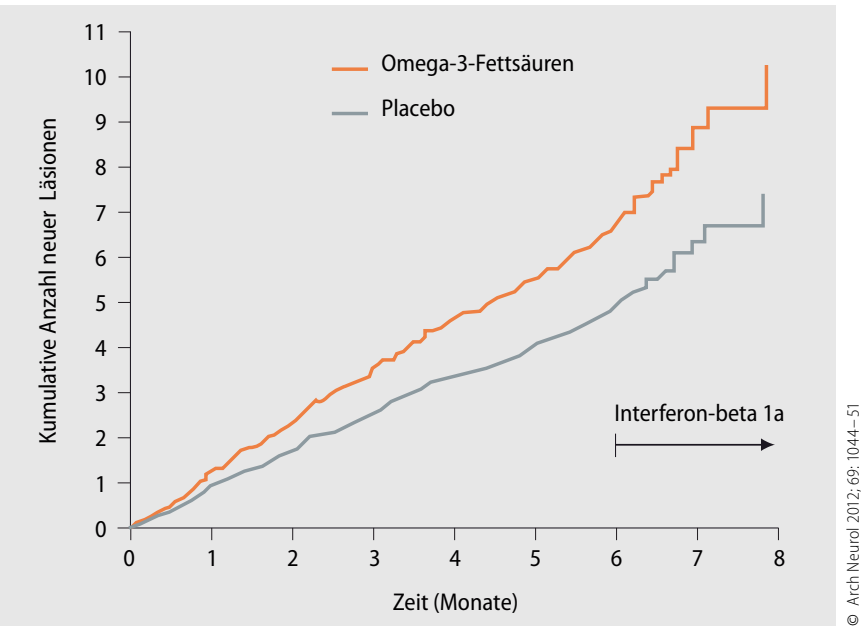

1 In der Omega-III-Fettsäuren-Gruppe traten sogar mehr neue $G d^{+}$-Herde auf, der Unterschied war jedoch nicht signifikant.

\section{-Kommentar von Prof. Volker Limmroth}

\section{Ende der Diskussion}

Eine Nahrungsergänzung mit Omega-3-Fettsäuren erscheint trotz umfangreicher populärwissenschaftlicher Literatur unwirksam. Auch wenn diese Studie nur mit 92 beziehungsweise 46 Patienten in jeder Behandlungsgruppe durchgeführt wurde, handelt es sich um eine wichtige Studie. Die inzwischen umfangreiche Literatur zur Nahrungsergänzung bei Autoimmunerkrankungen, insbesondere bei der MS sorgt bei Patienten immer wieder für große Unsicherheit. Auf populär wissenschaftlicher Basis wird ferner häufig der Eindruck vermittelt, dass allein durch die Änderung der Ernährung eine deutliche Reduktion der Erkrankungsaktivität oder gar Heilung möglich sei. Dies zwingt Neurologen immer wieder in lange Diskussionen über das Für und Wider von Nahrungsergänzungsmitteln mit verunsicherten Patienten. Die Studie wird nun hoffentlich dazu beitragen, für Patienten mehr Sicherheit im Hinblick auf das wichtige Thema Ernährung zu schaffen und wird vielen Kollegen helfen, langwierige Diskussionen über die richtige Ernährung mit Hinweis auf diese Studie abzukürzen.

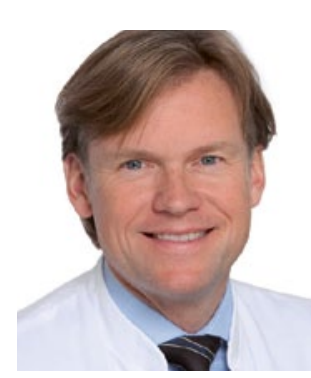

Prof. Dr. med. Volker Limmroth, Köln

Chefarzt der Klinik für Neurologie und Palliativmedizin Köln-Merheim E-Mail: LimmrothV@kliniken-koeln.de 